\title{
Semiconductors with a loop of extrema
}

\section{Citation}

Rashba, E.I. 2015. "Semiconductors with a Loop of Extrema." Journal of Electron Spectroscopy and Related Phenomena 201 (May): 4-5. doi:10.1016/j.elspec.2014.10.002.

\section{Published Version}

doi:10.1016/j.elspec.2014.10.002

\section{Permanent link}

http://nrs.harvard.edu/urn-3:HUL.InstRepos:23927493

\section{Terms of Use}

This article was downloaded from Harvard University's DASH repository, and is made available under the terms and conditions applicable to Other Posted Material, as set forth at http:// nrs.harvard.edu/urn-3:HUL.InstRepos:dash.current.terms-of-use\#LAA

\section{Share Your Story}

The Harvard community has made this article openly available.

Please share how this access benefits you. Submit a story.

Accessibility 


\title{
Semiconductors with a Loop of Extrema
}

\author{
E. I. Rashba \\ Physical Institute of the Academy of Sciences of Ukrainian SSR, Kiev, USSR
}

\begin{abstract}
Crystals with a Wurtzite-type structure are used as an example to show that owing to spin-orbit coupling a new type of the band structure can arise. For this band structure the extrema are reached at a circle, a loop of extrema, rather than in isolated points of the Brillouin zone. Specific properties of such semiconductors are studied theoretically, in particular, the peculiarities of the cyclotron resonance at low temperatures. In strong magnetic fields, spin-orbit coupling results in absorption at the frequency of electron spin resonance but driven by the electric vector of electromagnetic wave (combined resonance).
\end{abstract}

PACS numbers:

Spin-orbit (SO) coupling can result in a specific band structure such that, in a reasonable approximation, the extremum is reached at a circle, "a loop of extrema", rather than in isolated points of the Brillouin zone (B.Z.). In particular, such a band structure can arise in the lattices of the wurtzite type $\left(C_{6 v}^{4}\right)$.

Analysis of the position of the potential zero-slope points of the energy spectrum in absence of spin-orbit coupling was performed for a lattice of the wurtzite type by using the general formulae of Ref. [1] that include only characters of irreducible representations. It shows that the zero slope can arise at an arbitrary point of the B.Z., having a shape of a right hexagonal prism, but at its top and bottom faces, and also at its vertical ribs $P$ for the states transforming according to the twodimensional representation. Remarkably, the only exclusion are the vertices $H$ of the prism for the states transforming according to the two-dimensional representation. Two bands can stick together in the point where an extremum is reached only at two instances, either when this happens in the point $H$ or at the symmetry axis $\Delta$ (for representations $\Delta_{5}$ and $\Delta_{6}$ ). In the latter case the constant energy surfaces have a shape of two unwarped ellipsoids of revolution, one embedded into the other. In other points of the B.Z. the bands are nondegenerate near the extrema.

If nondegenerate extrema are reached in $P$ or $\Delta$ in absence of spin-orbit coupling, then with the $\mathrm{SO}$ coupling included the dispersion law reads

$$
E(\mathbf{k})=a k_{\perp}^{2}+b k_{z}^{2} \pm \alpha k_{\perp}, \text { where } k_{\perp}=\sqrt{k_{x}^{2}+k_{y}^{2}}
$$

( $z$-axis is parallel to the hexagonal axis c). The parameter $\alpha$ is proportional to the SO coupling strength. Energy $E(\mathbf{k})$ reaches its minimum [Footnote 1: All presentation below is in terms of the conduction band.] at a circle of a radius $k_{0}=\alpha / 2 \alpha$. The depth of the loop, i.e., the energy separation between the point $\mathbf{k}=0$ and the bottom of the band, is equal to $\varepsilon=\alpha^{2} / 4 a$. At $E<0$, constant energy surfaces have the shape of tori. If the terms of the higher order in $\mathbf{k}$ are taken into account in $E(\mathbf{k})$, the loop disintegrates and minima of $E(\mathbf{k})$ are achieved in a set of isolated points of the B.Z. However, the saddle points between them should be low and therefore the ap- proximation based on the loop of extrema is expected to turn more useful than the description in terms of isolated points of extrema. Similarly, the splitting of each of the $\Delta_{5}$ and $\Delta_{6}$ bands by SO coupling results in two energy bands. In one of them $\left(\Delta_{9}\right)$ the slope vanishes at $\mathbf{k}=0$, while in the other $\left(\Delta_{7}\right.$ or $\left.\Delta_{8}\right)$ a loop of extrema develops [2].

The data on intermetallic compounds [3] suggest that $\varepsilon$ may be large enough to make the displacement of the minimum from a symmetry axis observable experimentally. Also, the current interpretation of the data on CdS [4] according to which both the bottom of the conduction band and the top of the valence band are reached in the center of the B.Z., and similarity between the optical spectra of CdS and a number of different crystals, suggest that in the crystals of this group the loop of extrema really exists. Therefore, a more detailed investigation of semiconductors with a loop of extrema is both of intrinsic interest and can serve as a convenient model for investigating the properties of noncentrosymmetric semiconductors.

In a magnetic field $\mathbf{H} \| \mathbf{c}$ the effective mass $m_{\text {eff }}$ of electrons diverges at the surface of the cylinder of a radius $k_{0}$ because $\partial E / \partial k_{\perp}=0$. The effective mass of electrons is positive, $m_{\mathrm{eff}}>0$, for $k_{\perp}>k_{0}$ and is negative, $m_{\text {eff }}<0$, for $k_{\perp}<k_{0}$. Therefore, inside the cylinder electrons behave dynamically as holes. At low temperatures, when charge carriers populate the region near the bottom of the band and the concentrations of "electrons" and "holes" are nearly equal, the absorption of the right- and left-polarized electromagnetic waves propagating along $\mathbf{c}$ should be nearly equal, the Hall constant should vanish [Footnote 2: At a total constant concentration of the carriers in the band], etc.

A detailed investigation of the resonances can be performed by using the exact solution defining two branches of quantized states

$$
E_{0}=\hbar \omega^{*} \beta, E_{s}=\hbar \omega^{*}\left(s \pm \sqrt{\beta^{2}+\gamma^{2} s}\right), s \geq 1,
$$

where $\omega^{*}=2 a e H / \hbar^{2} c, \gamma=2 \sqrt{\varepsilon / \hbar \omega^{*}}, \beta=\beta_{0} H / \hbar \omega^{*}$, and $\beta_{0}$ is an effective magnetic moment of the electron. The probabilities of quantum transitions driven by coupling of the electron charge to the electromagnetic wave 
can be calculated using two-component eigenvectors that are conveniently expressed in terms of the eigenfunctions of a harmonic oscillator. One can check that the selection rules are $s \rightarrow s \pm 1$, and both the intraband and interband transitions are allowed [5].

Let us begin with intraband transitions. When $E_{s} \gg \epsilon$, the resonance condition is $\omega \approx \omega^{*}$, which corresponds to the usual pattern of the cyclotron resonance (CR) for electrons with ellipsoidal constant-energy surfaces. On the contrary, when $E_{s}<0$, frequencies of the transitions between adjacent energy levels strongly depend on $E_{s}$. Therefore, when $k_{\mathrm{B}} T$ is less or of the order of $\varepsilon$ but is larger than the separation between adjacent energy levels, the transitions frequencies are different for different groups of electrons and therefore no distinct resonance is expected. The most intriguing peculiarities of the CR should manifest themselves in the ultra-low temperature region when $k_{\mathrm{B}} T$ is less than the level separation and the majority of electrons populates the lowest energy level. In this parameter range, as it follows from Eq. (2), the resonance frequency becomes an oscillating function of $H$ (if the field $H$ is not too strong). Then, under the usual conditions of the experiment with $\omega$ kept fixed and $H$ changing, and for a favorable relation between $\varepsilon$ and $\hbar \omega$, the resonance is achieved at several values of $H$ (with alternating resonances in the right and left waves). These peculiarities of the $\mathrm{CR}$ originate (i) from the existence of a negative effective mass region, $m_{\mathrm{eff}}<0$, and (ii) due to a non-monotonic dependence of $E_{s}$ on $s$, and might be encountered also in other similar systems.

Interbranch transitions are of special interest in the strong magnetic field region. When $\beta_{0} H \gg \sqrt{\varepsilon E_{s}}$, the frequency of quantum transitions is $\omega_{0} \approx 2 \beta_{0} H / \hbar$, as it follows from Eq. (2). Obviously, $2 \beta_{0} H$ is the energy of the electron spin flip. It is natural to term such a resonance (i) driven by the electric vector of an electromagnetic wave but (ii) possessing the frequency of a paramagnetic resonance controlled by the electron spin-flip energy as a combined resonance (COR) [5]. This resonance is weaker than the CR by a factor of the order of $\varepsilon / \beta_{0} H$, but is of a few orders of magnitude stronger than the conventional paramagnetic resonance driven by the coupling of the electron magnetic moment to the magnetic vector of electromagnetic wave. In physical terms, the mechanism of COR is absolutely transparent: a strong external field breaks the SO-coupling and controls the frequency of spin flips, while the SO-coupling makes possible the spin-flip transitions driven by the Lorentz force (with a probability decreasing with $H$ ). Obviously, the COR may manifest itself also in different noncentrosymmetric semiconductors.

Magnetic susceptibility $\chi$ also shows a rather peculiar behavior [6]. It tends to its $\varepsilon=0$ limits both (i) for a nondegenerate electron gas at high temperature and arbitrary field and (ii) for a strong field at arbitrary temperature. At low temperatures and weak fields, the susceptibility is positive, $\chi>0$, and does not depend on the temperature. The paramagnetic sign of the susceptibility originates from the fact that in weak fields the lower Landau levels sink under the bottom of the energy band, as is seen from Eq. (2). At $T=0$ the monotonic part of $\chi$ is a singular function of the chemical potential $\zeta$. For $\zeta<0$ [Footnote 3: The same origins are chosen both for the chemical potential and the energy.] it is positive and increases with $\zeta$ monotonically, at $\zeta=0$ it has a pole of the order $\frac{1}{2}$ and changes the sign, and for large $\zeta$ it tends to the standard expression for a simple band. Characteristic beats are superimposed onto the quantum oscillations of the susceptibility. They originate from the existence of two close Fermi surfaces.

Properties of semiconductors with a loop of extrema in a magnetic field parallel to the plane of the loop have also been investigated.
1 E. I. Rashba, Fiz. Tverd. Tela 1, 407 (1959).

${ }^{2}$ E. I. Rashba and V. I. Sheka, Fiz. Tverd. Tela, Collection of Papers, v. II, p. 162 (Acad. of Sciences of the USSR Publishers, Moscow-Leningrad, 1959).

3 F. Stern, J. Phys. Chem. Solids 8, 277 (1959).

4 R. N. Dexter, J. Phys. Chem. Solids 8, 494 (1959).
J. L. Birman, Phys. Rev. 114, 1490 (1959).

D. G. Thomas \& J. J. Hopfield, Phys. Rev. 116, 573 (1959).

${ }^{5}$ E. I. Rashba, Fiz. Tverd. Tela 2, 1224 (1960).

${ }^{6}$ I. I. Boiko and E. I. Rashba, Fiz. Tverd. Tela 2, 1874 (1960). 